OBSERVATIONS

ON

\title{
THE NECESSITY AND METHOD
}

or

FURTHER INVESTIGATING

THE

DISTINCTIONS BETWEEN SYPHILIS

AND

OTHER VARIETIES OF VENEREAL DISEASE.

By R. WELBANK, Esq.

Read March 13, 1827.

IT would seem a sufficient plea for soliciting the attention of this Society to the subject of venereal diseases, that they are maladies of every day occurrence, in which human suffering, moral as well as physical, is often deeply involved, and in which our judgment is sought with the greatest anxiety, and our treatment contemplated with the most vigilant distrust. But it may be further urged, that accumulating records are now generally known to have rather exhibited the extent of our uncertainty upon these subjects; than diminished those difficulties and doubts which confound the student, and perplex the experienced. 
There exists indeed at present so little unanimity among the members of the medical profession as to the nature of venereal diseases, that the state of opinion may be termed a schism, in which the one party is inclined to believe in the distinct plurality of primary poisons, the other to refer the great diversity of venereal disease to modifications of one virus by varieties of individual constitution. To add to the complexity of practice attendant upon these sentiments, many have still further suggested a progressive alteration in the maladies themselves, some are content to credit an indefinite combination with scurvy and scrofula, and others secure themselves against the possible contingencies of empirical treatment, by the supposition of a mercurial disease, equally indeterminate, and as easily and generally applicable in every difficult emergency.

Of these hypothetical changes and combinations, not observable in other contagious diseases, the consideration may be reasonably deferred, at least till we are something more agreed in prescribing the limits and characters' of the original venereal disease, or of those several affections which are said to have become composite. It is moreover superfluous to infer any such alterations in the nature of venereal diseases, for various records, of various dates, from various countries, shew that the same complexity has always and every where attended their history. Lastly, mercury can only be accused 
with justice of exasperating under certain circumstances some forms of venereal disease, especially the phagedænic. This, however, may not unfrequently occur in identical characters, without any possibility of its being ascribed either to the use of mercury, or to venereal contact.

In considering the individuality of the several, or the unity of all venereal diseases, if we admit constitutional modifications of actions resulting from specific stimuli, of such potency as to be the exciting cause in one instance of a disease directly and uniformly tractable to the influence of mercury, and in another of a disease even exasperated by the use of the same remedy, we may at once, as far as practical rules are concerned, disregard the limitations of nosology, and leave treatment to individual empiricism. It will however be safer at present to subscribe to "the old evasion of occult causes," or, in plain words, admit our fallibility, than suppose that natural processes so variously and capriciously deviate from general laws. We have sufficient and determinate difficulty to en. counter, without the indefinite addition of hypotheses, which the doctrine of anomaly must supply.

All admit that there is a great variety of morbid affections of the genitals, and of symptoms consecutive or attendant on them. All again will admit that many of these, multiform as they are, occasionally resemble each other so nearly, prima facie 
and for a varying period or stage, as to be undistinguishable by the most experienced. All, or at least those who have witnessed the irreparable ravages of these diseases, too often promoted by inappropriate treatment, will readily testify to the utility and even necessity of diagnosis.

Inoculation, in the varieties most important from their contamination of the general system, is an unjustifiable mode of investigation in the present state of our knowledge : nor if we had the aid of inoculation, could we hope to establish that uniformity of effect so necessary in questions of causation, for some of the most highly contagious forms of venereal disease may arise without any specific excitement, and might therefore confound even the experiments of inoculation. How then can we prosecute inquiry? How distinctly ascertain the several processes of disease induced by the several venereal poisons, if they be several?

Difficulties, which are apparently almost insuperable, seem still to leave us a practicable method of investigation. Instead of recording with laboured minuteness the resemblance or dissimilarity, confessedly sometimes fallacious, of primary sores, of eruptions, or of other really or seemingly consecutive diseases in the cases of different individuals, we should faithfully chronicle the diversity of disease existing at the same time in the same person. We should note, for instance, the various character 
and progress of a phagedænic sore, as it attacks different tissues, or the phenomena of several of these sores, when they have occurred at the same time, in different situations, from the same infection. Let us also record the multiform secondary effects of the same disease, contemporaneous in their appearance, or co-existent in the same system, and various as they are manifested in absorbents, mucous membrane, skin, cellular tissue, fibrous membrane, or in the bones. From repeated observation of collective phenomena, we shall soon arrive at the inference that many affections, often noticed in conjunction, but various in their apparent characters, are in reality the constant result of one or other distinct stimulus acting upon a diversity of organization. By a patient and unbiassed prosecution of this mode of inquiry, we cannot fail soon to acquire diagnostic data, which will enable us to solve some of the most difficult problems in the distinction of venereal complaints, for there will be readily found some forms, or some stages of disease in each so characteristically peculiar, that by them we may safely consolidate classification and regulate treatment.

Let any one observe for himself the following contrasted points of general difference between Syphilis and the Phagedænic disease. These are two forms of venereal disease, most important from the general utility, and (as I am compelled to believe). occasional necessity of employing mercury 
for the former, and of restricting, if not altogether discontinuing its use in the latter, except as a local agent. They are also important as indistinguishably simulating each other prima facie under some circumstances, or in some states or stages of their several symptoms. They are important, as they may perhaps yet be found to be the only two forms capable of contaminating the circulation, and they are not the least important that one of them, the Phagedænic disease, although contagious, and consequently justly classed with venereal affections, is, in all its symptoms, not unfrequently spontaneous, or consequent on simple excitement. Inattention to these latter facts has too often aggravated the sufficient evil of physical suffering by moral imputations.

Syphilis in general is first observed at a more remote period from sexual intercourse than other venereal diseases. It commences in some point of inflammation, which is soon characterized by abrupt induration. It proceeds, sooner or later, to indolent and uniform ulceration of the surface, between which and the edge there is no undermining groove of separation. The inguinal glands are little, and often not at all disturbed by the absorption of the virus, even when secondary diseases have already manifested themselves.

The secondary diseases of Syphilis arise in the series noted by Mr. Hunter, attacking first the 
skin and throat, and then deeper-seated parts. They are ushered in by a soft and much accelerated pulse, without any other obvious febrile symptom, and by severe nocturnal pains. They are indolent, and slow of progress. They consist of an eruption of firm and slightly elevated spots, from which pellicles or scales are from the commencement successively detached. These spots are thick about the scalp, chin, forehead, and upper and inner part of the thighs. In situations where there is hair, they frequently form slightly-elevated crusts of a pale colour. When they appear on the palms of the hand, or soles of the feet, they are characterized by a thick honey-comb desquamation of the dense cuticle. They are more disposed to superficial ulceration, when confluent or situated between opposed and secreting surfaces, as the angles of the mouth, scrotum and thigh, the nates, or between the toes. The ulceration of the tonsil is attended with little pain at first, and excavates the part affected deeply and often in a triangular form, as if the tonsil were split. This ulceration slowly acquires, especially on the supervention of simple excitement, a smooth buffy surface. Syphilitic nodes are a late symptom, and appear in the form of a painful, but chronic enlargement of the bone affected. Syphilis is a rare disease, and is in every form directly superseded pro tempore by the excitement of mercurial irritation. It is long intractably stationary, or even progressive in most cases, unless mercury or sarsaparilla be employed.

voL. XIII. 
Phagedana may arise spontaneously in cachectic habits, either in its primary or secondary forms. Sometimes it is first noticed many months after sexual intercourse, sometimes on the day following; it exhibits in general a rapid inflammatory ulceration, succeeding to pustule, extensive excoriation or fissure of surface. Frequently there is puriform discharge from the urethra. This is often record. ed, under the name of Gonorrhea, as the cause of secondary diseases. It is however in many cases only a concomitant mark of a certain cachectic con. stitution. It sometimes first appears contemporaneously with the supposed secondary diseases, sometimes three or four weeks after primary ulcerations of the genitals. Sometimes it recurs many months after supposed cure, in conjunction with eruptive diseases. It differs both in progress and cure from simple gonorrhea, and may have appeared ten or twelve times in one patient, from constitutional lia. bility. Primary phagedænic ulcerations are various and multiform, and do notalways exhibit a continued disposition to extend themselves. They in general spread at their edges, in parts or the whole of their circumference, and are characterized by considerable tumefaction of cellular tissue, and frequently phimosis. Natural processes of reparation in milder forms of the disease quickly follow those of destruction. The secretions in phagedænic ulceration are, for the most part, sanious or purulent, and ordinarily profuse. The inguinal and other absorbent glands become subject in many cases to rapid 
enlargement and suppuration. These affections of the absarbents often arise when the exciting sores are healing or have healed.

The constitutional diseases of Phagedæna are frequently coexistent, and even simultaneous, in their origin with those termed primary. They do not attack different structures in any regular series, and even nodes are contemporaneous with cutaneous and other diseases, or even precede them. Some of them often exist in an active state, with little disturbance of the circulation, and are generally characterized by rapid or sudden local inflammations. The affections of the skin consist of distinct purplish or brownish spots, with or without elevation, of a thick papular, vesicular, and pustular eruption on the trunk and extremities, the three forms being generally conjoined. These eruptions are not found on the palms of the hands and soles of the feet, and exhibit an appearance of desquamation on their decline only. Sometimes there are distinct superficial tubercles slightly vesicating, or large phlyzaceous pustules forming crusts. When the phagedænic virus attacks the cellular tissue, it causes deep red and much elevated tubercles, of various sizes and furunculous character, the larger of which often exhibit a considerable extent of phlegmonous inflammation. A superficial and rapid ulceration of phagedænic character exposes the sloughy cellular tissue, which leaves a deep cavity on its separation. Frequently may be noticed a 
combined affection of the skin and cellular tissue, attended with superficial ulceration, which extends at its edge, while it heals in its centre, forming an islet or peninsula of skin. These sores, which are frequently covered with prominent crusts of rupia, resemble in shape the base of the horse's foot, and leave a white depressed cicatrix, circular or oval, and very characteristic of phagedænic ulceration. Sometimes large patches of integument become red and indurated, and subsequently ulcerate superficially into many circular, oval, and crescentic sores. These are especially frequent about the carpus and tarsus, and round the elbow, ancle, and wrist joints, and on the thigh and calf of the leg. The phagedænic ulcerations of the throat and fauces suddenly occupy a considerable space, exhibit often a shreddy irregular surface, and a bright red and. everted edge. Sometimes the phagedænic process is limited to the mucous membrane, which is grooved out by the advancing edge, while processes of reparation are visible in the parts first affected. Suddenly suppurating inflammation occurs, even at early periods, about and beneath the periosteum of various bones, particularly of the cranium, nose, and tibiæ, and about the carpus and tarsus, this symptom being more generally observed where mercury is indiscreetly employed. Intense and agonizing pain attacks the external parts of the head, in many cases. With the eruptions of the skin is often joined iritis. Inflammation and ulceration of the larynx, enlargement of the testicles, effusion 
into the joints, especially of the knee, and phagedænic ulceration about the lacrymal sac and eyelids, may be added to this long catalogue of disease; and sometimes there exists extensive herpetic ulceration of the skin of the face and forehead. This affection often destroys in its progress the alæ and septum of the nose.

Mercury administered so as to affect the circulating system is superfluous in the milder symptoms, and exasperates generally the more severe. Even when apparently yielding to an increased action of mercury, the maladies suddenly relapse, and are often protracted in their duration. The disposition of many of the milder forms of this disease to processes of natural cure and reparation, is a frequent source of error in estimating the utility of mercury, and even the more severe forms are in some instances, for a time, superseded by pushing, as it is termed, a mercurial course. Each relapse, however, of disease, especially in its more formidable shapes, is aggravated in severity proportionably to the disturbance of the constitution unnecessarily induced by such injurious treatment, till at length an irremediable state of disease is established. Iritis is the only affection in which the risk of irreparable injury by delaying the use of mercury, counterbalances the almost certain evil of administering it so as materially to disturb the circulating system. 
$\therefore$ The classification, in the above sketch, of the triple eruption of papulæ, vesicles, and pustules, with the symptoms of the phagedænic poison is, I am aware, not altogether accordant with the observations of Mr. Carmichael, who candidly admits that he has not met with many instances in which he was enabled to follow the primary ulcer of his pustular venereal disease to its constitutional symptoms. He has moreover stated that this variety forms " the natural link between the papular and phagedænic venereal diseases." That the papular eruption (which even Mr. Carmichael's cases, as well as those published by $\mathrm{Mr}$. Rose in the Transactions of this Society, shew to be seldom purely papular) is not a consequence of common gonorrhea, general experience would as clearly indicate, as that all discharges from the urethra are not the same in their nature. That it does not succeed to the elevated ulcer, better named venerola vulgaris, the evidence of $\mathrm{Mr}$. Abernethy may be adduced, who says, after a most accurate portrait of this form of disease, "I never saw any secondary symptom succeed to this species of ulcer." During many years attention to the phenomena of this very common sore, no instance has occurred to my own observation or knowledge of secondary disease in such cases treated either with or without mercury. There are, however, sores both primary and secondary, (the result of the phagedænic poison manifested 
in other attendant symptoms, ) which closely. resemble the venerola vulgaris. They are perhaps in general more superficial, larger, and more rapid, but both in venerola and phagedæna processes of reparation commence in the centre, so that these, sores occasionally nearly simulate each other; Lastly, having repeatedly seen the papular, vesicular, and pustular eruption conjoined with symp: toms unequivocally the effect of the phagedænic virus, I am compelled to consider it one of the milder consequences of the same stimulus.

- The proposition of referring a great variety of disease to one or other distinct poison, is neither hypothetical nor suggested merely by the accumulating evidence of my own note-book, but may be confirmed by collation of the records furnished by Mr. Abernethy, Mr. Carmichael, Mr. Rose, Mr. Evans, and others, whose opinions are often in wide variance on the subject of these maladies, Nor can I dismiss this part of the subject without expressing a belief that if we content ourselves with recording collectively the various symptoms induced by the same stimulus in the same individual, but in different parts and tissues, we shall find that the most intricate complexity of venereal disease will gradually assume something like order, and that the eye alone will soon detect essential distinctions in the great majority of phenomena.

It remains for us to examine the arguments for 
the supposition of an inextricable combination, or rather confusion, of these complaints. The facts which have induced some to attribute to one source diseases not only so various in their primary characters, so different in their progress, so distinct in the production or non-production of multiform secondary affections, but even tractable by the most opposite treatment, are principally, that different sores or other primary disease in one or different individuals have been observed to succeed to infection from the same female. For in. stance, Dr. Hennen has mentioned that after connexion with one woman in the same hour, one man escaped without disease, a second contracted chancres and elevated sores, and a third gonorrhea. And it has been further noticed that various forms of secondary eruptions and other maladies have arisen from the same primary sore in the same individual, or from sores, precisely similar in apparent character, in different individuals. And lastly, that the same eruptions or other secondary diseases have attended or followed sores roidely dissimilar in their apparent characters.

A solution of the former difficulty relating to the complexity of primary venereal disease, is readily found in the obvious fact, that the same poison will give rise to different phenomena in parts differently organized. The virus of gonorrhea affecting the urethra produces gonorrhea; if it invades the cellular tissue of a torn frænum 
or an abraded surface, it may produce the venerolic ulcer. This sore alone, in addition to other occasional peculiarities, exhibits four totally distinct appearances in its several stages. But it is said, true chancre, real syphilis, has arisen under the same circumstances of infection. What then are the prima facie phenomena by which we should venture to determine syphilis? Mr. Hunter has testified to his occasional perplexity, and acknowledged that he has seen sores putting on "all the appearance of a chancre," under circumstances precluding the possibility of real syphilis. Mr. Abernethy has described several varieties of true chancre, and has directed his observations against diagnosis, founded on prima facie appearances of venereal diseases. Mr. Samuel Cooper in an article in his Surgical Dictionary upon this subject, valuable from its collective information as well as impartiality, has expressed his concurrence with the opinion of Mr. Guthrie, that many of the ulcers produced from the matter of gonorrhea "will occasionally assume every character of chancre, and cannot be distinguished from it." There is, indeed, a too generally repeated and most fallacious description of a real chancre, viz. a sore circular or oval, abruptly indurated, covered with adherent matter, having no disposition to reparation or progressively ulcerating. Not only is there in this portrait no single characteristic individually applicable to syphilis, but the collective phenomena are not unfrequently seen in 
other ulcerations of the genitals, and especially in the venerola for some weeks. In the treatment of this latter disease, I have repeatedly seen in my own practice a free use of mercury enjoined, and subsequently discontinued from its inutility or prejudicial influence, by several of the first surgical authorities of this metropolis.

But were it granted that syphilis had arisen in the same individual, together with the venerolic ulcer under the same circumstances of infection, rather than reason generally from such an excep. tion or adopt so unphilosophical a conclusion, as that one and the same cause acting under precisely the same circumstances, could produce effects so distinctly different as venerola and chancre, the one disease being directly amenable to mercury, and the other often exasperated by its use; it would be safer to suppose that the virus of syphilis had co-existed in the infecting person. We are not justified, indeed, in inferring from the absence of disease the non-existence of a poison, as it is quite possible that a poison may have existed, from whose effects the original bearer was still exempt. Various instances of sores resulting from connexion with women apparently healthy must be familiar to surgeons; venerola and phagedæna are in the number of these. A point not suffi. ciently adverted to in considering the multiplicity of disease, apparently arising from the same infection, is the disposition which may.exist in different 
or the same individuals to spontaneous morbid affections of the genitals, and consequently not unlikely to succeed the mere local excitement of sexual intercourse. Among these it may be use: ful to notice the psoriasis præputii, and scrotalis, in which may frequently be observed distinct spots of a brownish tint and elevated. These are often scaly, and with them may exist similar spots thick about the scalp and upper extremities, especially at the wrists. There are also in some instances erythematous and aphthous inflamma. tion of the tonsils and fauces and mouth. Dis. charge from the urethra, in general of short duration, occurs repeatedly in persons subject to these affections.

A source of great variety in the effects of morbific poisons, is that various degree of power which is ascertained by direct experiments to be proportionate to the temporary activity of the disease from which the contagious matter is taken; This fact is of high importanee, both as regards secondary as well as primary symptoms; for it may, perhaps, depend on this various degree of virulence whether an eruption in the same textures of the skin shall be papular, vesicular, or pustular, or a phagedænic sore be deep or superficial, stationary or disposed to extend its ravages.

- And lastly, in our view of the sources of com. plexity in the multiform phenomena of the same 
poison; we have still to include the many adventitious circumstances which may tend by their stimulant or sedative agency to produce a corresponding influence, or the prima facie characters of primary venereal disease.

On the subject of the diversity of secondary diseases, accumulating observations seem unnecessarily perverted to an increase of our perplexity rather than of our knowledge. This has arisen, as in the details of primary disease, from deciding upon their nature, from the present appearance, so often fallacious, of eruptions, \&c., instead of their history and progress and their concomitant affections. Resemblance or dissimilarity are converted into identity or total difference. Yet Dr. Bateman has said, "These eruptions assume such a variety of forms that they bid defiance to arrangement according to their external character, and in fact they possess no common or exclusive marks by which their nature and origin are indicated. There is, perhaps, no order of cutaneous appearances, and scarcely any genus or species of the chronic eruptions, which the secondary symptoms do not occasionally resemble."

The occasional ca-existence of distinct primary diseases is a possible origin of much complexity in the secondary phenomena. Not however to dwell on these or other causes of diversity applicable to the secondary as well as to the primary 
diseases, let us examine what proof can be adduced from them that the position of Mr. Carmichael, that there are distinct poisons productive of peculiar and distinct effects, is not in the main correct.

Various eruptions and other disease, it is said, have arisen in the same individual from the same sore, or in different individuals from sores precisely similar in their apparent characters; and lastly, the same forms of disease have succeeded to sores widely dissimilar in appearance. With regard to this last position, it has been shewn, and is generally admitted, that sores widely dissimilar in appearance may result from the same infection. It cannot therefore be argued that these secondary diseases were not the product of one. and the same poison, because the primary sores were unlike each other. If, again, as observation will constantly shew, various forms of eruptions and other secondary disease arise in the same individual from the same sore, à fortiori the diversity may be expected in different individuals whose primary sores may be admitted to have been even the same. The question therefore resolves itself into the simple inquiry, whether, because various effects are seen in various tissues, resulting from one virus, and because some of these closely simulate primâ facie the effects ascribable to other poisons, we are justified in confounding them altogether? 
The object of this paper is not to enter into. complicated details, but to suggest reasons for a patient investigation of the opimions of Mr. Carmichael, to the general truth and accuracy of which my own experience compels me to offer a humble, but very grateful testimony. If investigation be prosecuted without prejudice, we cannot fail to ascertain the distinct individuality of the syphilitic and other paisons. We shall then be able to consolidate a nosology, or rather nomen-. clature, already diffuse beyond the ordinary capacity of the human mind. Treatment will be further simplified by the united observations of physicians and surgeons, and a common classification of these maladies adopted. At present, the nomenclature of many is not more diverse than the remedies, for while ecthyma cachecticum and rupia simplex or prominens, are treated with: tonics or lenient remedies by the physician, the same affections under the common name of venereal disease are often condemned to a very in. jurious influence of mercury by the surgeon.

If it be said that diagnosis is unimportant, my own observation will vouch that irreparable injury of the palate and of the penis, and even the sacrifice of life have resulted from unfounded apprehensions of employing mercury in the cure of syphilis. Of the exasperation of phagedænic disease by the indiscreet use of the same remedy, three fifths of the venereal patients admitted into a 
London Hospital afford a sufficient evidence. This latter disease is, to use the words of $\mathrm{Mr}$. Carmichael, " a malady which is chiefly rendered formidable by injudicious interference, and which too often leads the victim of mal-practice through a disgusting and offensive train of symptoms to a painful and lingering death."

The original object of the preceding communication was, as stated, not to investigate the too extensive subject of venereal disease in detail, but rather to suggest certain topics for further inquiry, with the view that the observations offered might be confirmed or corrected by a more extended examination than the limited experience of any in. dividual could effect. The practical utility however of illustrative cases having been suggested; the following records are appended as exhibiting some points in the three more important varieties, syphilis, phagedænic venereal disease, and the venerola vulgaris of Mr. Evans. It would have been perhaps better to have considered these fully and separately at some future period, but no regret on my part will attend the imperfection of anticipated publication, if any additional impulse is given to that inquiry, which the frequency, complexity, and severity of venereal disease so imperatively demand.

Primary Syphilis is in general distinguishable by its character of abrupt cartilaginous induration; 
slow progress, and superficial ulceration. It is in these, and other points already considered, different from ulcers, which, commencing in pustular inflammation, are for a while rapidly progressive, and become indurated during the excitement of their earlier stages. It seems questionable whether pus is a product of syphilitic action. The secondary symptoms of syphilis are, as before observed, peculiar. Mercury affecting the constitution, even slightly, directly and uniformly antagonizes and supersedes the influence exerted by the syphilitic virus, but is no specific antidote to the virus itself; which must be gradually modified or eliminated by the system at large*. Hence, if the most severe and protracted courses of mercury be discontinued while the virus still exists in the system, the dis. ease rapidly recurs on the subsidence of the mer. curial action, nor does the most violent use of the remedy afford more immunity from relapse than the mildest, if adequate to the pro tempore subversion of the disease. On the other hand, the disease recurs with a severity proportionate to the super.

* Mr. Hunter has adverted to the occasional inadequacy of courses of mercury, and suggested an explanation in the supposition of a disposition and subsequent action, of which the latter only is curable. Among early records of the failure of an empirical use of mercury, is the history of a charlatan, who is stated by Benvenuto Cellini to have acquired great reputation by his ointment, but would have been massacred, had he remained in Rome till the event of his treatment could have been more fairly estimated. 
fluous derangement of health, occasioned by abuse of the remedy.

It is an important question how far secondary diseases are capable of renewing or increasing the quantity of virus in the system by fresh absorption. It is a fact, that the absorbents become affected subsequently to the establishment of secondary disease; for instance, those behind the ear and at the side and back of the neck, when the'scalp and throat have been attacked by secondary disease. As mercury has in syphilis the property of converting an ichorous into a puriform secretion, and inducing processes of healthy reparation, to protract the formation of fresh virus is attended with unnecessary hazard. The most obstinate and tedious cases of syphilitic infection appear to be those in which diseased actions, whether primary or secondary, are allowed to proceed. The consequences resulting from the adoption or neglect of these principles; as well as the practical value of a possible spontaneous cure of real syphilis, will be illustrated by a few cases.

T. N. had observed extensive excoriation three weeks before, which for the most part healed readily, but left abrupt induration about the frænum præputii. This gradually increased, and became raw and superficially ulcerated. It was determined, in consultation with another surgeon, to effect a slight action of mercury upon the patient's system, voL. XIII. Q Q 
and the plan was adopted for about three weeks. The disease readily yielded, and had disappeared in a few days. In about two weeks from the discontinuance of the remedy, slight soreness of the throat was noticed, and continued progressive for four weeks at the sea side. At this period he had manifestly excavated ulcers of the tonsils, scaly eruption, nocturnal pains, accelerated pulse, and the hair was falling off. He now consulted, on his return to town, a third surgeon, by whom the use of sarsaparilla alone was suggested. The symptoms yielded in about three weeks, but relapsed in about a fortnight after discontinuance of this plan of treatment. It was now determined to try a severe use of mercury for eight weeks; the syphilitic symptoms immediately yielded. The patient after this course went to the sea side, but returned within twelve days from the period of omitting mercury, with deep sloughy ulcer of the left tonsil, and greatly disturbed health. Sarsaparilla decoction again mitigated the symptoms, but the disease relapsed, and became actively progressive. The extension of the malady was deemed a sufficient warrant for a repetition of a severe use of mercury, which plan was vigorously prosecuted about ten weeks. The patient became well rapidly, and continued free from disease till about three weeks after discontinuance of the treatment, when he again returned from the sea side with extensive disease of the pharynx and tonsil, and with incipient inflammation and ulceration in the centre of the soft 
palate. The disease being now pronounced to be mercurial, sarsaparilla, bark, and some local treatment, was ineffectually tried. The patient's sufferings increased: the extent of the diseased surface could not be seen, the soft palate was perforated and sloughing, the general health was sinking rapidly, and ptyalism, from irritation in the throat, supervened in a distressing degree.

The use of sarsaparilla was now sanctioned by a fourth opinion, and the employment of mercury still delayed. Within two days the disease had advanced so close to the uvula as to threaten a division of the palate. Seven grains of the hydrargyrum cum creta, with one-third of a grain of opium, were now administered twice a day, and in a few days the quantity was increased to ten grains. In three days the diseased processes were checked, the sloughs partially separating, and the ptyalism, subsiding. The patient's health and strength were gradually reestablished, and all tendency to relapse of disease occasionally manifested by a few spots, or slight soreness of the throat, were directly and effectually remedied by a few additional doses of compound calomel pill, which medicine was continued in small quantities to the final and complete restoration of his health. About fifteen months elapsed during the treatment of this case. The aperture in the palate remained unclosed. 
M. L. observed a sore spot, rather thickened, on the integuments of the penis, about three weeks after sexual connexion. This gradually increased, and two other points of inflammation assumed a similar character of tubercular disease, superficially ulcerative. Simple dressings and external cold were recommended. Under this treatment the extent and bulk of the diseased parts slowly increased till, at about eight weeks from the period of infection, febrile excitement with pains supervened. In consultation with an eminent surgeon, a mild course of mercury was recommended. A very freiquent and soft pulse now quickly regained its natural state, and the syphilitic symptoms, local and constitutional, promptly receded. The mouth was slightly affected during six weeks' inunction. In five weeks after the discontinuance of the mercury the patient had sore throat, scaly eruption, disturbed health, and his former frequent and soft pulse. The use of mercury was re-enjoined in consultation, and adopted to slight ptyalism for four weeks, with the prompt effect of curing his several complaints. Notwithstanding the advantage of seaair, and the use of sarsaparilla, he returned with incipient ulceration of the tonsil within two weeks fiom the discontinuance of the mercurial treatment. He was now fortunately content to prosecute a mild mercurial treatment, which speedily reinstated him in health, and being continued for some weeks, was cautiously discontinued when it appeared that he was no longer obnoxious to his former com- 
plaints. Small doses of compound calomel pill were adequate in this case to the restoration and maintenance of health for the necessary period.

H. L. contracted a tubercular chancre at the side of the frænum, much indurated and superficially ulcerative. The disease yielded to an internal employment of mercury, which slightly affected his gums, and was soon discontinued from its prejudicial influence on his general health. In about ten days after the omission of the remedy, he had a buffy excavated ulcer of the tonsil, which soon became actively progressive under antiphlogistic and palliative treatment. He was admitted into a public institution. Considerable ptyalism had supervened on the increasing disease of his throat, and probably led, with other circumstances of his case, to treatment with decoction of sarsaparilla only. Considerable mitigation of symptoms attended at first the use of this remedy, but the disease actively relapsed, and destroyed the patient by its unchecked ravages, within eight weeks from its first attacking him.

M. E. contracted a large excoriated sore behind the corona glandis, which had healed under the use of mercurial pills continued to a slight affection of the gums, and the local employment of black wash. 
He soon became affected with pains in the limbs, which yielded to a renewal of small doses of mercury and diet drink. At about ten weeks subsequent to his first infection, he had still, while under the latter treatment, considerable induration left behind the corona glandis, encrusted spots in the beard, circular spots on the hands and feet, which had recently desquamated, ulcerative rhagades between the toes, and had had slight sore throat. These symptoms all yielded promptly to slight mercurial action, which had restored him to apparent health in about four weeks. He presented himself casually to my notice about five weeks after, and his symptoms had recurred, and were progressively getting worse under neglect.

L. E. contracted a sore near the frænum, which healed under the use of blue stone, but left induration. In about four weeks he had severe nocturnal pains, soon followed by slight sore throat and inflammation of the ear. This latter symptom was succeeded by discharge from the meatus of a reddish sordes, and deafness. Under the use of warm baths, aperient medicine, and low diet, the whole of these maladies became worse during about twelve days, and the small induration of the prepuce had increased to a bulky and raw sore. They now speedily yielded, including the deafness, to a mild internal use of mercury, which slightly affected the 
mouth. The disease subsequently relapsed, on the too early discontinuance of the remedy, which being resumed again, restored him to health.

P. E. contracted extensive excoriation, attended with much indurated thickening behind the corona glandis. During the subsequent six months this disease healed and reulcerated, under trivial and occasional local treatment. During the whole period the patient's health became progressively disturbed, and he fancied himself labouring under rheumatism. At length the primary disease became more actively inflamed, and mottling of the skin, scaly spots, and sore throat, were added to severe pains, which were worse at night. These symptoms all subsided rapidly on the speedy excitement of slight mercurial action, and the patient maintained his health for three months by alterative doses of mercury, which in no way prevented his attention to his ordinary occupations. He had no further relapse.

C. N. contracted a sore which did not attract notice till four weeks after connexion, at which period an indurated and excoriated tubercle evinced symptoms of active inflammation, and soon threatened sloughing. The inguinal glands on one side became tender and enlarged. All these affections 
yielded directly to the use of mercury by inunction. Nocturnal pains, scaly eruption, and soft and frequent pulse, indicated the contamination of the system soon after the discontinuance of the mercurial treatment. These symptoms were readily controlled by doses of compound calomel pill, administered according to circumstances, and the patient's health was gradually and completely reestablished by the prosecution of this plan of treatment, which neither interrupted business or pleasure, even during the severity of the winter months.

I cannot conclude the considerations relative to the treatment of syphilis, without expressing a conviction that it will be found uniformly and readily tractable to a rational and innocuous use of mercury. Unfortunately the surgeon has too often to contend with the patient's prejudices either "against the imaginary consequences of this remedy," or (still worse) in favour of some supposed specific powers, if it be employed in large quantities. Against this fallacious notion of "the safe side, and good old plan," a protest should be entered by every one acquainted either with the older or more recent documents on the subject of venereal diseases.

The following cases exhibit at least the probability of some intimate connexion between the pa- 
pular and pustular and the phagedænic venereal diseases, as well as the possibility of their spontaneous origin in some instances, and consequently of their constitutional as well as contagious nature. A collation of the history of papular and pustular venereal disease, as described by $\mathrm{Mr}$. Carmichael, with the history of papular eruptions by Mr. Rose, in the eighth volume of the Transactions of the Society, and the observations of Mr. Evans on the venerola superficialis, will shew a concurrence of opinion as to the coexistence of certain and many secondary phenomena distinct from syphilis, yet bearing close resemblance, in some forms or stages, to this disease, but not collectively. The same circumstance will be manifest by the most cursory comparison of facts relative to sloughing, erratic, and phagedænic diseases, as detailed by $\mathrm{Mr}$. Abernethy, Mr. Ferguson, Mr. Carmichael, Mr. Evans, and others.

I. G. had contracted a superficial ulcer, of the size of a sixpenny piece, situated at the side of the frænum. It commenced in a pustular form. For this he had taken a scruple of blue pill daily, and applied red precipitate. His mouth was made sore fourteen days, and the sore healed in a month. $\mathrm{He}$ had shortly severe pains, especially in the large joints, slight sore throat, and much fever. He repeated the use of mercury with aggravation of his symptoms, and a general eruption of papulæ, which became pustular in their progress, inflammation of 
the eyes; and sore throat., These symptoms super. vened while his mouth was sore, and declined gra. dually. The severe pains and fever shortly recurred, and did not yield readily to treatment with diet drink and extract of conium, but gradually subsided, and irregularly relapsed during a period of two months. His health was finally re-established by five weeks' residence in the country, the beneficial influence of the change of air being directly manifest.

E. G. wife of the preceding patient, had intercourse with her husband a month after the healing of his sore, but at a period when his health was much disturbed by pains, \&c. In a few days she observed discharge, unattended by local pain or irritation, severe pain in the head, shoulders, wrists, and knees, and in about seven days more a crop of pimples, which soon formed yellow heads. A third succession of these fell under my notice, and was accompanied by a rather foul ulcer of the right tonsil and palate, of a very inflammatory character. She blew a foul discharge from her nose, and had severe pains in her joints, and much fever. Diet drink and tartarized antimony were prescribed during a fortnight. The symptoms, with the exception of the ulcer of the throat, gradually improved. The irritation in the throat had, at this period, so increased that medicines were swallowed with great difficulty. The detail of symptoms and 
treatment during three subsequent weeks, shews little benefit from treatment, and a capricious variability in the symptoms. The disease of the throat had extended itself considerably, and exhibited the foul surface of the phagedænic forms of ulceration. She was now admitted an in-patient of a public institution, and a free use of mercury, together with local fumigation, was commenced. At first there seemed some improvement; as the mouth became sore, the throat partially exhibited a clean surface. After eleven days she was salivated. The partial improvement and the salivation continued after the omission of the mercury by inunction at this date. At the termination of the fourth week from her admission she had died, apparently by extension of disease to the larynx. One crop of eruption, in the first case, did not proceed either to vesicular or pustular disease, but exhibited convex distinct spots, for the most part formed by an aggregation of papulæ, and desquamating after about three weeks. This variety is constantly confounded with the syphilitic psoriasis, and nearly resembles indurated acne.

P. N. observed discharge from the urethra three weeks after intercourse with a female; an inguinal gland subsequently became enlarged. These symptoms yielded to pills (taken three times a day), which made his mouth sore. In two weeks he had a thick eruption on the skin of pimples and pus- 
tules. These got well without medicine, in two successive crops. He had afterwards a more protracted eruption, with sore throat and large circular sores, of which the cicatrices are visible on the thigh. At the period of his case falling under my observation, he had a thick copper-coloured eruption, with partial desquamation, and a large patch of inflamed and thickened skin near the ancle, ulcerating in crescentic patches superficially. These were getting well under a severe action of mercury, but were marked by the copper tint long after their decline, a point in which they much differed from real syphilis.

A gentleman's servant, who had had no venereal disease for six years, and then only discharge from the urethra, after suffering disturbed health and vague pains about the chest, became covered with a dense papular and pustular eruption. The spots were various in colour, from bright red to dark chocolate, the more recent elevated considerably; some almost petechial in appearance; he had considerable fever. These symptoms yielded to simple treatment, but relapsed repeatedly. In addition to them was subsequently noticed swelling about the ancles, with tenderness and swelling of the periosteum of the tibia. Diet drink, with compound calomel pill and country air, were the most beneficial measures. 
L. S. contracted a sore of the prepuce, commencing in a crack or fissure of the skin, the day after sexual intercourse. This continued to get worse under the action of mercury for some time; was not healed at the period of connexion with another female : this last intercourse was succeeded by discharge from the urethra. The sore now spread rapidly, and at the period of my seeing him was about the size of a half-crown, fungous in the centre, and phagedænic at its edge. He had sore throat, pain and soreness of the chest, and dense pustular eruption about the arms. A subsequent crop of eruption about four weeks after the preceding, exhibited large convex spots, like the syphilitic psoriasis, but desquamating only on their decline, and interspersed with evident pustules.

A. N. contracted ulceration and discharge from the urethra. These symptoms appeared two months after sexual intercourse. The sore commenced in pustules, and spread with considerable swelling of the prepuce; a bubo suppurated; his mouth was not affected by mercury during the cure. About seven months from the date of infection (to which period he had remained well), he had sore throat, successive crops of papulæ and pustules, severe pains, especially about the head, and a renewed discharge from the urethra, without any recent sexual intercourse. 
M. E. exhibited well marked phagedænic sores on the face, arm, and legs. He had never had any primary sore on the genitals, but ten or twelve attacks of discharge from the urethra.

H. N. never had either discharge or sore of the genitals. No cicatrix could be seen on the genitals. Had resided in the East Indies with evident detriment to his health. During the last ten months had been more or less ill, and subject to phagedænic sores of the skin; has at the time of my seeing him, well marked phagedænic sores on the arm. These became well from the centre, under linseed poultice, the exhibition of aperient medicine, and the local use of some stimulating lotion.

M. Y. an Irish pauper, has well marked phagedænic eruption with ulcerated throat and very disturbed health. Neither threat of consequences, nor promises of relief, could induce him to acknowledge that he had had any venereal affection of the genitals for twenty years. There were no cicatrices.

S. R. had been treated in the preceding year for noli me tangere: the disease did not yield till 
mercury was employed. A crescentic portion of the left ala nasi was destroyed; the angle of the mouth exhibited the cicatrix of a sore, which had spread superficially. At the period of my seeing him he had a large patch of inflamed and thickened skin over the elbow joint, on the surface of which were many circular and crescentic ulcerations of a manifestly phagedænic character. He had had no venereal disease of the genitals for nearly twenty years.

My limits will not allow me to trespass on the Society, by detailing cases of herpetic or erratic ulceration of the skin and mucous membrane, which seem to me a milder variety of that state of constitution in which the more severe forms of phagedænic disease occur, and are not unfrequently seen in conjunction with them. Such cases are in general tractable by escharotics or stimulants, the decoction of sarsaparilla, and good air. Cinnabar fumigations and the yellow wash exercise a very beneficial influence on many cases of phagedænic disease. To the melancholy records of the more severe forms described by Mr. Carmichael, I could add much in confirmation, but nothing of practical importance. I cannot however dismiss this part of the subject, without adverting to the endemic diseases supposed to be in many instances contracted " by eat- 
ing or drinking out of the same utensils with those labouring under them."*

The history of sibbens and yaws collated with documents relating to scurvy, would tend much to shew the constitutional origin of many of the more appalling varieties of the same disease. Much is certainly chargeable on the severe employment of mercury in the phagedænic venereal disease. One of the most protracted cases which have fallen under my observation occurred after discharge from the urethra, unattended with ulceration, and had been four times treated with courses of mercury, one of which was extended to five weeks' salivation. This treatment was often attended with temporary benefit, but eventually left the patient with multiplied and exasperated symptoms, no longer amenable to the same remedy. That the malady, in its worst forms, is not always attributable to injudicious use of mercury, may be conjectured from the loss of the nose, so frequently noticed among the South Sea Islanders, in Captain Cook's Voyages. The instances of real syphilis invading the bones of the nose are rare, if any. None have occurred to my own observation.

* See an account of the Sibbens of Scotland, \&cc. Bell on the Venereal Disease, Vol. II. p. 438. 
The following cases may perhaps tend to illustrate the distinct nature of the venerola vulgaris. That this form of disease is at least not very likely to be a source of secondary disease, would appear from the circumstance of its being the product of the same infection as simple gonorrhea; from its being metastatic with discharge from the urethra; and from its occurring repeatedly in the same individuals. I have seen it in six or seven instances in the same person. There is however a mild pustular variety of phagedæna, forming an ulcer nearly resembling in appearance, and even undistinguishably, the processes of venerola, especially in its elevation of edge and central granulation. Contamination of the system is not unfrequently consequent or attendant upon this form, which seems to be closely connected, if not identical with the ve. nerola superficialis described by Mr. Evans*.

H. M. contracted three well-marked venerolæ, one on the integuments of the penis, and two near the edge of the prepuce : in cutting some dressing, which he had just applied to the two last, he accidentally wounded the glans penis, and thus subjected himself to a fresh inoculation from the juxtaposition of the sores on the foreskin. He subsequently inoculated a cut-finger in the act of mictu-

* See Remarks on Ulcerations \&c. p. 93, and note. voL. XIII. 
rition, and as this last infection was soon attended with much local suffering and pain, extending up the arm, and in the axilla, he consulted another surgeon. He was put under the action of mercury for two weeks, with aggravation of his symptoms, and considerable tumefaction of the finger affected. Six leeches were applied to the finger, and three of the punctures, included with the ulcer in a bread and water poultice, were also infected. The mercury was discontinued, and the diseases gradually commenced and completed their reparative processes. No secondary symptoms supervened.

B. S. had been using mercury three weeks, and had affected his gums during that period, for a sore behind the corona glandis, which exhibited the appearance of venerola vulgaris. The surface was red, but the edge still sharp and inflamed. He assured me that the sore began to spread, if he discontinued the mercury, and the local use of argenti nitras, which fact he had probably noticed during the ulcerative stage of the disease. The malady readily began to heal on the omission of his former expedients, and was followed by no secondary symptoms.

H. E. had scratched off a small wart near the frænum, and in the site of it soon observed a small 
yellow ulcer. As this went on increasing, and did not after two weeks exhibit any processes of reparation, he became anxious as to the propriety of delaying mercury. By the advice of another surgeon, he now used mercury by inunction, with the effect of retarding the disease already established, while a fresh ulcer in the immediate vicinity continued to extend itself. Both sores healed by the processes peculiar to venerola vulgaris, on the discontinuance of the mercury, and were not succeeded by any secondary symptoms.

W.N. shewed me a well marked venerola behind the corona glandis, of which the appearance had exhibited no change for the preceding eight or ten days. He had used mercury for a month, and his gums were still affected. The ulcer was red on the surface, but sharp-edged, and with no disposition to cicatrization. Healing processes soon succeeded to the omission of mercury, and the use of simple aperient medicine. He had no secondary symptoms. This gentleman had subsequently at a distant period indurated syphilitic chancre, which, with its secondary effects, readily yielded to the use of mercury.

S. $H$. had venerola vulgaris on the dorsum and glans penis, in three well-marked specimens. He R R 2 
had subjected himself to two courses of mercury without advantage. He recovered readily at the sea side, after discontinuing his former treatment, and had no secondary symptoms.

I have selected the preceding cases, as they occurred at too remote a date to leave any question as to the possibility of any secondary symptoms, and because they exhibit the inutility, if not the prejudicial influence of mercury in general. It is however just to observe, that small doses of calomel and opium, or other preparations of mercury, are frequently beneficial where venerolæ, after the completion of their ulcerative stage, are indurated and fretful. 